Reprod. Nutr. Dévelop., 1982, 22 (1 B), 171-178.

\title{
Gliose de myélinisation et glioses réactionnelles au cours du développement du nerf optique chez le rat
}

\author{
J. FULCRAND, J. VALAT, A. PRIVAT $(*)$
}

Laboratoire de Neurophysiologie, Université des Sciences, 34060 Montpellier Cedex. (*) Laboratoire de Culture de Tissu nerveux, INSERM U 106, 92150 Suresnes.

Summary. - Myelination gliosis and reactive gliosis in the developing optic nerve of the rat.

Our radioautographic results obtained with light and electron microscopy confirmed that the oligodendrocytes are the only cells involved in the formation of the myelin sheath in the developing primary visual system of the rat. The oligodendrocyte, whose differentiation requires the intervention of an axonal signal, causes myelination gliosis, allowing, among other things, the nervous influx to acquire its definitive speed.

Radioautographic and freeze-fracture analyses of Wallerian degeneration in the optic nerve after enucleation showed the proliferative modalities of cells issued from the glioblastic precursors involved in reactive gliosis. The microglial cell participated in the process of phagocytosis (resorption gliosis) aided by the astrocytes that formed the glial scar (substitution gliosis). In this context of dynamic neurobiology, it is now possible to study the role of glio-axonal interactions in the regeneration of the CNS using the transplanted nerve technique.

\section{Introduction.}

La plupart des études concernant le développement du système nerveux central chez les mammifères se rapportent aux neurones. Les cellules névrogliques quantitativement importantes ont été considérées jusqu'à ces dernières années comme des éléments accessoires, cantonnées aux fonctions de soutien, trophique et métabolique du tissu nerveux. Les travaux plus récents (Mori et Leblond, 1969 ; Rakic, 1971 ; Fulcrand et Marty, 1973 ; Privat, 1975 ; Skoff et al., 1976) mettent l'accent sur d'autres rôles dévolus aux différentes lignées gliales, liés aux processus maturatifs du système nerveux central chez les mammifères.

Dans ce contexte, les voies visuelles primaires, en particulier le nerf optique, constituent un support approprié pour l'analyse des glioses de myélinisation et réactionnelles, en raison de leur structure cordonale dépourvue de corps cellulaires. Ce système sensoriel permet d'autre part une approche expérimentale aisée, 
axée sur le comportement des cellules névrogliques impliquées dans la gliogenèse et la dégénérescence wallérienne du système nerveux central (Fulcrand et Privat, 1977).

\section{Matériel et méthodes.}

Des rats Sprague Dawley ont été énucléés à des stades-repères choisis en relation avec la myélinisation des fibres nerveuses : $2,5,8$ et 20 jours après la naissance. Pour chacun de ces stades, les délais de survie s'échelonnent entre 1 et 200 jours. Préalablement au sacrifice, certains de ces animaux avaient reçu une injection unique de thymidine tritiée. Des blocs de nerf optique prélevés du côté ipsilatéral et controlatéral à la lésion, ces derniers servant de témoins, ont été préparés pour les microscopies optique et électronique ainsi que pour l'autoradiographie. La technique de cryofracture a été également utilisée afin de mieux connaître les stades précoces de la dégénérescence wallérienne.

\section{Résultats.}

Les résultats obtenus sur le double plan qualitatif et quantitatif montrent les modalités réactionnelles et prolifératives des différents types névrogliques, glioblastes, astrocytes, oligodendrocytes et cellules microgliques.

Les glioblastes (fig. 1 D) apparaissent comme les précurseurs des lignées gliales. Ils présentent une forme généralement allongée avec un rapport nucléoplasmique élevé et sont dépourvus de prolongements. Ils constituent 40 p. 100 de la population chez l'animal témoin pendant les 15 premiers jours postnataux. Chez les animaux énucléés à 2, 5 et 8 jours (fig. 2 B) ce pourcentage décroît rapidement à 10 . En revanche, chez les animaux opérés à 20 jours, le nombre de glioblastes négligeable au départ connaît un léger pic de l'ordre de 5 p. 100 une semaine après. En raison de la réduction du compartiment glioblastique, le nombre absolu de glioblastes marqués par la thymidine tritiée est notablement appauvri par rapport à l'animal témoin, tandis que l'index de marquage est augmenté. Cela traduit une activité mitotique intense des quelques glioblastes présents. Ces 2 modalités plaident en faveur d'une transformation rapide des glioblastes.

FIG. 1. - Autoradiographies de coupes semi-fines colorées au bleu de toluidine. L'éclairage mixte fait apparaître les grains d'argent sous forme de points brillants.

FIG. 1 A. - Animal énucléé à 2 jours ; délai de survie : 8 jours. La flèche indique un astrocyte marqué (A) avec son noyau encoché et le nucléoplasme clair. Gr. 1280.

FIG. 1 B. - Animal énucléé à 8 jours ; délai de survie : 3 jours. Oligodendrocyte marqué (O) montrant le nucléoplasme et le cytoplasme foncés. Gr. 1280.

FIG. 1 C. - Animal énucléé à 20 jours ; délai de survie : 5 jours. Deux astrocytes marqués (A) à nucléoplasme clair. Gr. 1280.

FIG. 1 D. - Animal témoin âgé de 10 jours. Glioblaste marqué (G) caractérisé par des amas de chromatime dispersés dans le noyau Gr. 1280. 

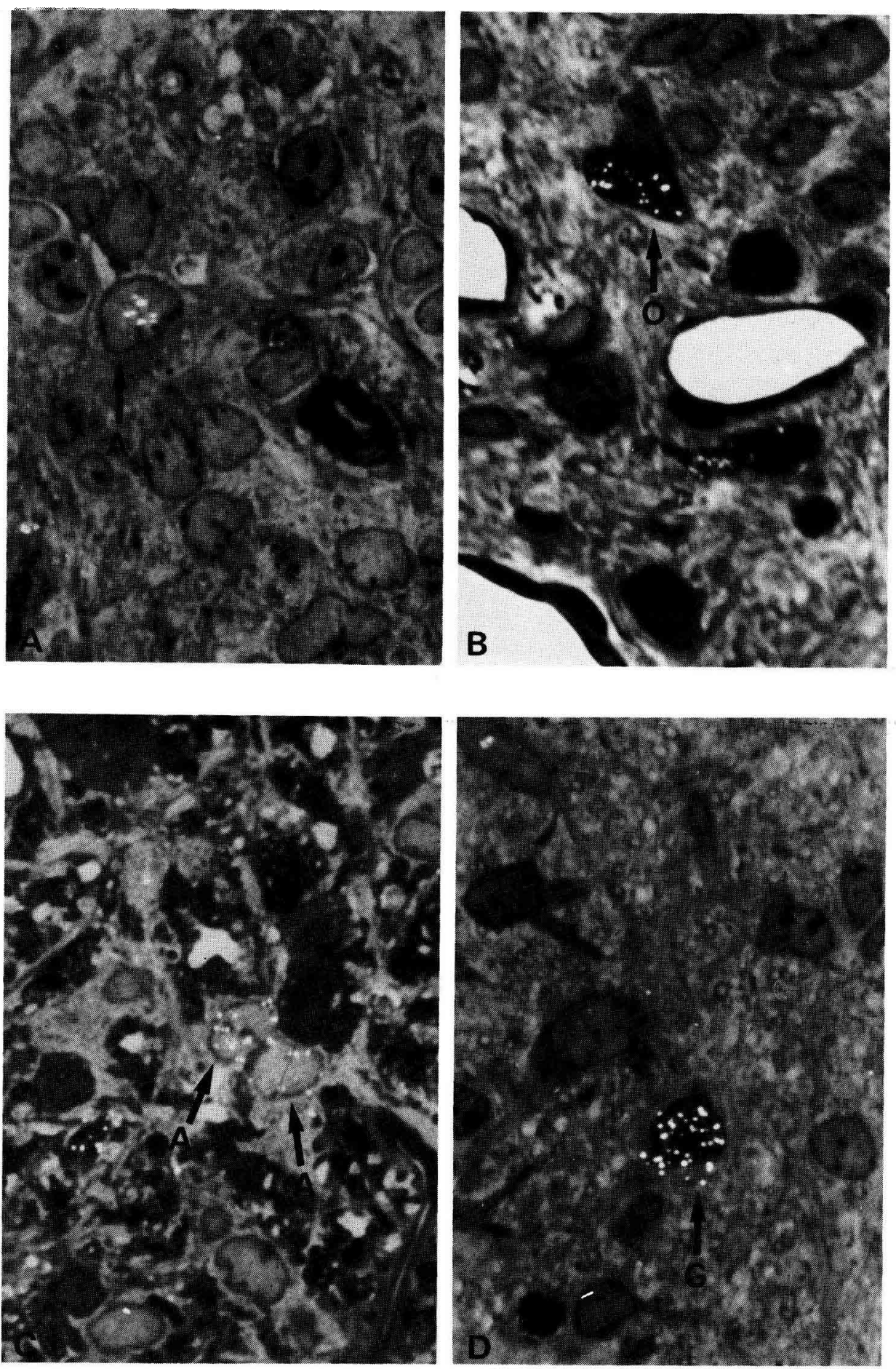
Les astrocytes sont caractérisés chez l'animal témoin par un noyau clair de forme régulière, à chromatine finement granuleuse condensée à sa périphérie ; le cytoplasme est également clair, riche en gliofilaments, et présente des citernes de réticulum granuleux à contenu dense (fig. $2 \mathrm{~A}$ ). Son contour est irrégulier, hérissé de prolongements remplis aussi de gliofilaments. Chez l'animal opéré (fig. $1 \mathrm{~A}$ et $1 \mathrm{C}$ ), le contour du noyau est plus échancré, tandis que le cytoplasme montre une densité plus forte de gliofilaments. Les pourcentages oscillent entre 60 à la naissance et 40 à partir du $15^{\mathrm{e}}$ jour chez l'animal témoin, tandis que chez les animaux opérés à 2,5 et 8 jours, ces valeurs se situent entre 70 et 90 p. 100, contre 50 à 70 p. 100 pour les animaux énucléés à 20 jours.

Les astrocytes se multiplient peu chez l'animal témoin. Après énucléation, un bref pic prolifératif précède une courbe d'évolution située à un niveau plus bas que chez le témoin. Or le compartiment astrocytaire augmente ; il est donc vraisemblablement alimenté par les glioblastes.

Les oligodendrocytes apparaissent dans le nerf optique vers le $5^{e}$ jour postnatal. Ils évoluent en 3 types successifs conformément au schéma proposé par Mori et Leblond en 1970.

Leur noyau est généralement plus dense que celui de l'astrocyte, ainsi que leur cytoplasme beaucoup plus riche en organites, citernes de réticulum granulaire empilées à contenu clair, Golgi plus ou moins hypertrophié et présence de microtubules, en particulier au niveau des prolongements rectilignes. Chez l'animal témoin le pourcentage s'accroît régulièrement jusqu'au $30^{\mathrm{e}}$ jour et se stabilise aux environs de 60 p. 100.

Chez l'animal énucléé au stade-repère 2 jours, le pourcentage d'oligodendrocytes essentiellement clairs reste inférieur à 5 . Aux stades-repères 5 et 8 jours (fig. $1 \mathrm{~B}$ ), un pic initial de l'ordre de 20 p. 100 est suivi d'une décroissance régulière. Enfin au stade-repère 20 jours, la courbe d'évolution des oligodendrocytes, en majorité sombres, passe de 60 à 20 p. 100. La plupart d'entre eux s'enkystent dans une gaine lâche de pseudo-myéline.

Chez l'animal témoin, l'activité mitotique n'est sensible qu'au $10^{\mathrm{e}}$ jour. L'index de marquage est relativement plus élevé chez les animaux énucléés.

Les cellules microgliques interstitielles sont reconnaissables chez le nerf témoin par leur noyau allongé, sombre et à contour irrégulier. Leur cytoplasme est peu abondant, dense, parsemé d'inclusions, avec de longues citernes de réticulum granulaire flexueuses; leurs rares prolongements sont courts et irréguliers.

FIGURE 2.

FIG. 2 A. - Microphotographie d'un nerf optique témoin chez un animal âgé de 20 jours. Astrocyte à nucléoplasme clair et homogène. Dans le cytoplasme s'observent des faisceaux de gliofilaments et des citernes de réticulum granulaire à contenu dense. Gr. 8450.

FIG. 2 B. - Autoradiographie électronique de nerf optique chez un animal énucléé à 8 jours; délai de survie : 7 jours. Cellules marquées à caractères immatures présentant essentiellement un cytoplasme riche en ribosomes. Gr. 11300. 

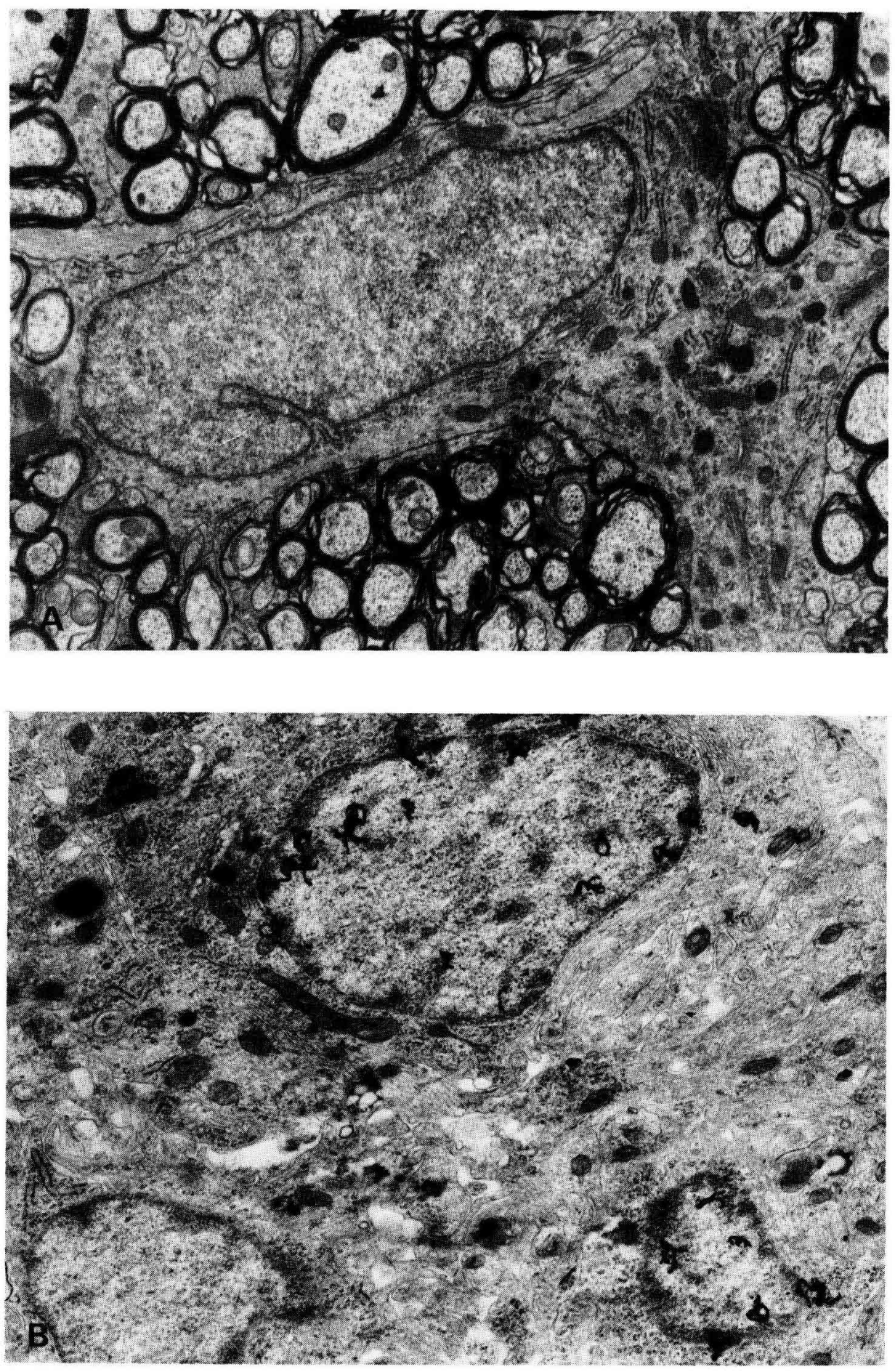
Elles constituent environ 5 p. 100 de la population névroglique. Chez l'animal opéré, 2 formes se succèdent, l'une sphérique chargée d'inclusions phagocytaires, l'autre plus allongée quiescente. Les valeurs se situent autour de 20 p. 100 aux divers stades-repères considérés avec un décalage progressif de leur réactivité.

Le marquage est négligeable chez l'animal témoin, et de l'ordre successivement de $5,5,20$ et 2 p. 100 aux 4 stades-repères. A chacun de ces stades les péricytes montrent un index de marquage plus élevé $(15,18,45$ et 2,5 p. 100). $\mathrm{D}^{\prime}$ autres cellules, hématogènes par exemple, se rencontrent aux stades précoces et au voisinage de la lésion (Vaughn, Hinds et Skoff, 1970 ; Imamoto et Leblond, 1978).

\section{Discussion.}

Les considérations découlant des résultats obtenus au cours du développement sur les multiplications des cellules névrogliques, qualifiées de glioses, se rapportent essentiellement aux aspects fonctionnels. Dans ce contexte, l'accent sera mis sur les interactions entre la glie et le neurone et les mécanismes qu'elles soustendent. La plupart des éléments gliaux dérivant des glioblastes, il est normal $d^{\prime}$ indiquer tout d'abord que ces derniers participent à l'équilibre dynamique des différents compartiments névrogliques. Selon les circonstances, les glioblastes vont se différencier en oligodendrocytes, astrocytes, ou éventuellement en cellules microgliques comme l'attestent les résultats radio-autographiques. La prolifération des glioblastes et leur différenciation subséquente sont sous le contrôle direct de I'axone (Privat et als, 1981 a).

En ce qui concerne l'oligodendrocyte, il intervient dans la formation de la gaine de myéline (gliose de myélinisation), permettant à l'influx nerveux d'acquérir sa vitesse définitive. La différenciation de l'oligodendrocyte est sous la dépendance de signaux axoniques qui se manifestent aux différentes étapes de son évolution morphologique et biochimique (Bhat et al., 1981). Dans ce contexte, l'utilisation de mutants dysmyéliniques, comme la souris Jimpy, aide à mieux comprendre la cinétique des interactions glio-axoniques (Privat et al., 1981 b).

L'astrocyte, quant à lui, assure la formation de la cicatrice gliale (gliose de substitution) particulièrement importante au cours de la dégénérescence wallérienne du nerf optique. Cette gliose réactionnelle est également sous l'influence d'un ou plusieurs signaux axoniques, induits pendant la désintégration de l'axone plus ou moins rapide selon les stades-repères considérés (Fulcrand et Privat, 1977 ; Valat et al., 1978). L'analyse de ces signaux impliquant la mise en œuvre des techniques immuno-cytochimiques est déjà entreprise. La fonction cicatricielle de l'astrocyte peut constituer l'obstacle majeur à la régénération des fibres du système nerveux central, comme le montrent les expériences de transplantations du nerf optique dans le nerf sciatique de souris normales et mutantes (Fulcrand et al., en préparation).

La cellule microglique enfin est principalement mobilisée dans la phagocytose des débris cellulaires. Elément essentiel de la gliose de résorption, elle peut être aidée par les astrocytes et éventuellement les oligodendrocytes peu différenciés, 
ainsi que par les cellules hématogènes. L'origine embryologique de la cellule microglique, exclusivement ectodermique ou mésodermique, ne peut être actuellement déterminée. Nos résultats plaident en faveur d'une origine double, dépendante des conditions expérimentales.

\section{Conclusion.}

Au cours du développement du nerf optique chez le rat, l'ablation du globe oculaire et la dégénérescence wallérienne qui s'ensuit ont permis de mettre en évidence un réseau complexe et dynamique d'interactions existant entre les cellules névrogliques et les axones. La glioblaste, cellule souche, paraît extrêmement plastique dans sa multiplication et sa différenciation liées à l'environnement tissulaire. L'oligodendrocyte est étroitement dépendant de l'intégrité de l'axone en ce qui concerne la multiplication de ses précurseurs surtout au cours de la période de pré-myélinisation, et sa maturation ultérieure. En revanche, les oligodendrocytes déjà différenciés peuvent survivre en l'absence d'axones. L'astrocyte est la cellule réactive par excellence, capable de phagocytose aux stades précoces de sa maturation, responsable de la cicatrisation aux stades plus tardifs. La microglie, cellule phagocytaire dont l'origine est multiple, intervient à tous les stades, sa réactivité étant proportionnelle à l'intensité de la dégénérescence.

7 Réunion du groupe Développement I.N.R.A., Nouzilly/Tours, 14-15 mai 1981.

Remerciements. - Les auteurs remercient M. C. Bavière et P. Sibleyras pour leur collaboration technique.

Ce travail a bénéficié de l'aide de I'INSERM (contrat $n^{\circ} 81.79 .113 .6$ ) et de la FRMF.

\section{Références}

BHAT S., BARBARESE E., PFEIFFER S. E., 1981. Requirement for non-oligodendrocyte cell signals for enhanced myelinogenic gene expression in long term cultures of purified rat oligodendrocytes. Proc. nat. Acad. Sci. USA, 78, 1283-1287.

FULCRAND J., MARTY R., 1973. Maturation postnatale du tractus optique chez le rat: identification et quantification de la névroglie sur la radioautographies. Exp. Brain Res., 16, 466-475.

FULCRAND J., PRIVAT A., 1977. Neuroglial reactions secondary to Wallerian degeneration in the optic nerve of the postnatal rat : ultrastructural and quantitative study. J. comp. Neurol., 176, $189-224$.

IMAMOTO K., LEBLOND C.-P., 1978. Radioautographic investigation of gliogenesis in the corpus callosum of young rats. II. Origin of microglial cells. J. comp. Neurol., 180, 139-164.

MORI S., LEBLOND C.-P., 1969. Electron microscopic features and proliferation of astrocytes in the corpus callosum of the rat. J. comp. Neurol., 137, 197-226.

MORI S., LEBLOND C.-P., 1970. Electron microscopic identification of three classes of oligodendrocytes and a preliminary study of their proliferative activity in the corpus callosum of young rats. J. comp. Neurol., 139, 1-30. 
PRIVAT A., 1975. Postnatal gliogenesis in the mammalian brain. Int. Rev. Cytol., 40, 281-323.

PRIVAT A., VALAT J., FULCRAND J., 1981a. Proliferation of neuroglial cell lines in the degenerating optic nerve of young rats. A radioautographic study. Neuropathol. exp. Neurol., 40, 4660 .

PRIVAT A., VALAT J., LACHAPELLE F., BAUMANN N., FULCRAND J., 1981b. Radioautographic evidence for the protracted proliferation of glial cells in the central nervous system of Jimpy mice. Brain Res. (sous presse).

RAKIC P., 1971. Neuron-glia relationship during granule cell migration in developping cerebeliar cortex. A golgi and electron microscopy study in Macacus rhesus. J. comp. Neurol., 141, 283-312.

SKOFF R. P., PRICE D. L., STOCKS A., 1976. Electron microscopic autoradiographic studies of gliogenesis in rat optic nerve. I. Cell proliferation. J. comp. neurol., 169, 291-312.

VALAT J., FULCRAND J., PRIVAT. A., MARTY R., 1978. Radioautographic study of cell proliferation secondary to Wallerian degeneration in the postnatal rat optic nerve. Acta neuropathol., 42, 205-209.

VAUGHN J. E., HINDS P. L., SKOFF R.P., 1970. Electron microscopic study of Wallerian degeneration in rat optic nerve. I the multipotential glia. J. comp. Neurol., 140, 175-206. 